\title{
Flight planning and flexible use of airspace in Free route airspace area
}

\author{
Martin Kodera \\ Department of Air Transport, \\ Faculty of Transportation Sciences, Czech Technical \\ University \\ Horská 3, Praha 2, 128 03, Czech Republic
}

\author{
Jakub Hospodka \\ Department of Air Transport, \\ Faculty of Transportation Sciences, Czech Technical \\ University \\ Horská 3, Praha 2, 128 03, Czech Republic
}

\author{
Martin Chleboun \\ Navigation Department, \\ Travel Service a.s. \\ K letišti 1068/30, Praha 6, 160 08, Czech Republic
}

\begin{abstract}
The paper summarizes changes in the flight planning caused by the introduction of Free Route Airspace Project and suggests possible measures needed to be adopted across the whole system in order to ensure military and civilian aircraft remain segregated in a way that is today ensured by the system of conditional routes. The paper suggests a possible solution in flight planning using existing flight planning tools provided by the CFMU.
\end{abstract}

Keywords - flight planning, free route airspace, conditional route, flight planning tool

\section{INTRODUCTION}

Recent developments in airspace design in Europe aim towards complete withdrawal of airways and using an airspace that is defined solely by waypoints of various nature. Such airspace tends to be more effective than the conventional routebased system but inevitably loses some of the means the old system provides in an area of adjusting the traffic flow and smooth cooperation between civil and military users of the airspace.

Currently a system of three types of conditional routes (CDR) is employed in order to ensure segregation of those two very different types of traffic, but with Free Route Airspace Project (FRAP) the CDRs will be withdrawn along with other routes. A different measure has to be taken in order to ensure such segregation. This measure has to provide flight planning tools for aircraft operators that ensures distribution of information about activated temporary segregated area (TSA) and temporary restricted area (TRA) in a clear way.

This article focuses only on operational air traffic (OAT) as it is the most common and most affected by changes in the route system and route availability.

\section{FLIGHT INEFFICIENCY}

The pursuit of the highest flight efficiency is based on an effort to minimise the costs and the negative effects of aviation on the environment. Flight inefficiency or horizontal en-route flight inefficiency is defined as a distance of the actual flown or planned flight divided by the great circle distance of the flight and is expressed as a percentage.

Based on which factor of the airspace we are trying to evaluate, we are measuring different distances:

- Shortest Unconstrained Route (SUR) is a shortest route between two points planned using the existing route network. SUR does not take into account limitations by CDR or Route Availability Document (RAD). This distance measures how effectively is the route network designed

- Shortest Constrained Route (SCR) is a shortest route between two points planned by using existing route network and taking limitations by RAD and CDR into account. This distance then measures how effectively is the route network designed with regard to the limitations set by flow management

- Filed Tactical Flight Model (FTFM) is a distance of the last flight plan filed by the operator. It has to be equal or higher than the SCR distance. FTFM might be longer because of effects of aircraft operator's decisions which might include overflight costs, weather forecast, overflight permissions, ending validity of the route, possible regulations and slots on the route and of course the fact that the shortest route might not be known to the operator 
- CPR flight model (CPFM) is the actual distance flown by the aircraft determined on the Consolidated Position Reports. This distance is usually shorter than FTFM because of operational measures applied by ATC - most common being direct flight to a point further on the route.

The EUROCONTROL goal for the year 2014 is that SUR inefficiency would be $2,7 \%$ and FTFM inefficiency would be $4,15 \%$. On those numbers it is clearly visible, that it is expected that the operators will submit routes $1,45 \%$ more inefficient than they could. The reasons for this occurrence are mentioned above.

Should fixed route network stay preserved, it is not desirable to adjust the inefficiency to $0 \%$ as that would suggest great circle routings between all airports and limited possibility of air traffic flow management and routing adjustments.

\section{FRAP}

The motivation to introduce FRAP to Europe is driven by several factors. The first one naturally being cost savings for the operators in a form of flight time savings and fuel savings. The other one is reduction of emissions of carbon dioxide and nitrous oxides, which has gained a massive importance over recent years.

FRAP uses, for flight planning purposes, only waypoints in a letter name form (5LNC), VOR, NDB, DME or latitude and longitude. The aircraft operators can use any number of points in order to plan the flight route, but they must use at least one waypoint at each FIR border the flight crosses, unless is this measure adjusted by a Letter of Agreement between the two countries/FIRs.

The specific goal for airspace design set by EUROCONTROL is to optimise horizontal en-route flight efficiency for the whole system. This indicator specifies, by how many per cent is the actual flight route longer then a great circle distance.

\section{En-route flight efficiency improvements from free route intitiatives}

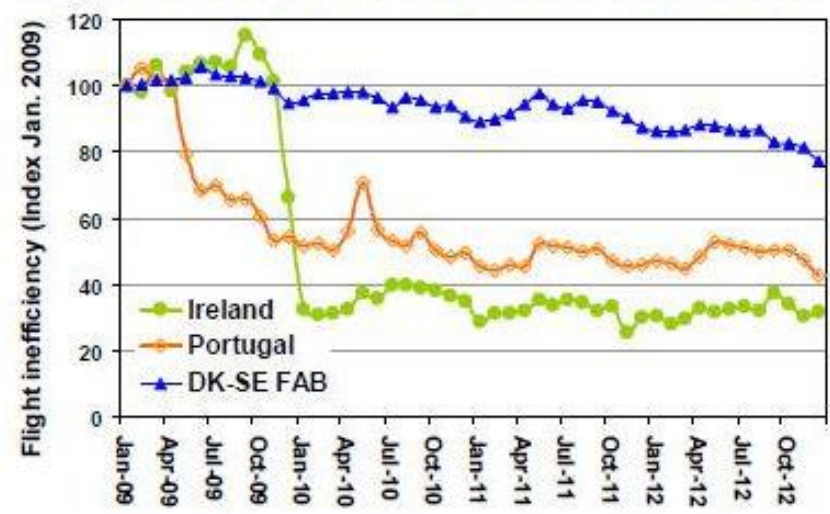

Figure 1. Flight inefficiency decrease linked to introduction of FRAP in Ireland, Portugal and FAB DK/SE Source: [2]

Based on the shortest routes available October 2013 during AIRAC cycle 1013, the shortest possible distances were determined for flights using the route network and for hypothetical flights using FRAP. A distance of 80NM has been deducted from each of these distances in order to omit the influence of departure and arrival procedures.

TABLE I. COMPARISON OF FLIGHTS USING FRAP AND EXISTING ROUTE NETWORK

\begin{tabular}{|c|c|c|c|}
\hline Flight & $\begin{array}{c}\text { Great circle } \\
(\boldsymbol{N M})\end{array}$ & $\begin{array}{c}\text { Route distance } \\
(\boldsymbol{N M})\end{array}$ & FRAP (NM) \\
\hline LKPR - EGLL & 484 & 501,6 & 486,4 \\
\hline LIRF - LKPR & 425,1 & 468,6 & 431,8 \\
\hline ENBR - GCTS & 2047 & 2128 & 2073 \\
\hline EYVI - EPKK & 260 & 294 & 272 \\
\hline
\end{tabular}

It is noticeable, that FRAP versions of the routes are significantly shorter, than the ones using conventional routes, which is also a reason, why FRAP or FRAP-like procedures, such as night directs or specified allowed directs, is widely introduced to the environment in Europe. This process is not centralized and the transition, or whether the transition will happen at all, rests entirely upon national ATS providers and local CAAs.

However it seems that soon the whole Europe will have FRAP or FRAP-like measures introduced, which raises questions of changes needed in order to ensure safe and smooth traffic flow without the route network and its predictability and managing possibility.

Some measures have to be introduced in order to ensure it will remain possible to activate the TSA or TRA on the pretactical or even tactical level (shortest activation period for these areas is 15 minutes) and make sure the aircraft operators will take appropriate measures and plan the flight clear of these areas, otherwise their flight plan will not be accepted.

Currently FRAP is introduced in Sweden, Denmark, Portugal and Ireland with northern Spain joining soon. Most of European countries are already planning to introduce FRAP or FRAP-like airspace.

\section{FLEXIBLE USE OF AIRSPACE}

Flexible use of airspace in a fixed route network is based on conditional routes, which are active or inactive based on an activation of the TSA or TRA. Once the CDR is closed, the operator cannot file a flight plan using this route and has to reroute the flight.

The CDR activation is published by an Airspace Management Cells (AMC) in a form of Airspace Use Plan (AUP) and Update Use Plan (UUP). All these messages from all AMCs are then added to Conditional Route Availability Message which is then used by the operators.

In the FRA area the AMCs have no way of adjusting the traffic flow and civil/military segregation by closing or opening routes because there are no routes left to be closed or opened.

The TSA and TRA are defined in a national AIP by vertical boundaries, stated in a flight level range in which the airspace 
is unusable and horizontal boundaries, which are usually a set of latitude and longitude coordinates marking the borders of an area. The problem with this way of marking the airspace is that the geographical coordinates used as horizontal borders have very little in common with the operator's desired route. And unless the operator has a sophisticated planning software allowing him to highlight the closed airspace they would have to sacrifice a lot of time and effort to determine if the desired route is limited by the closed area or not. Considering there are more than one TSA/TRA near the desired route, the planning of the flight might be very time consuming without appropriate aids.

However, the ability of the Network Manager, represented by Central Flow Management Unit (CFMU), to exclude OAT from certain areas that are reserved for a special use remains crucial. Doing so by publishing a list of unavailable directs is not a suitable solution because the number of direct routes between any two points crossing activated airspace would be tremendous.

The aircraft operators need a complex planning tool that would help them find a suitable, shortest possible routing under given condition by the airspace management and the operator as well. along with a message showing in which part of the validity check the error has been found. For logged in IFPS users, which means all stakeholders, a route suggestion tool is also available, allowing the operator to identify the city pair and list of compulsory overflight points or FIRs or list of denied FIRs and points, and offering an IFPS compliant route for requested time and date of the flight as well as the desired flight level. The date time and flight level are taken from the FPL that is being validated.

\section{FRAP PLANNING SUGGESTIONS}

Considering the existence of Europe-wide Free Route Airspace and a withdrawal of the whole route system in Europe, a problem rises for flight planning purposes and for flexible use of airspace. Along with withdrawal of the routes, conditional routes are withdrawn as well. This puts a major obstacle to the FUA concept because no instrument of excluding civilian traffic from a certain area would exist anymore.

The TRAs and TSAs would not cease to exist and neither would the military traffic. A new measure has to be taken in order to inform the operators of an active area, rejects a flight plan whose route does not avoid the area with a given margin

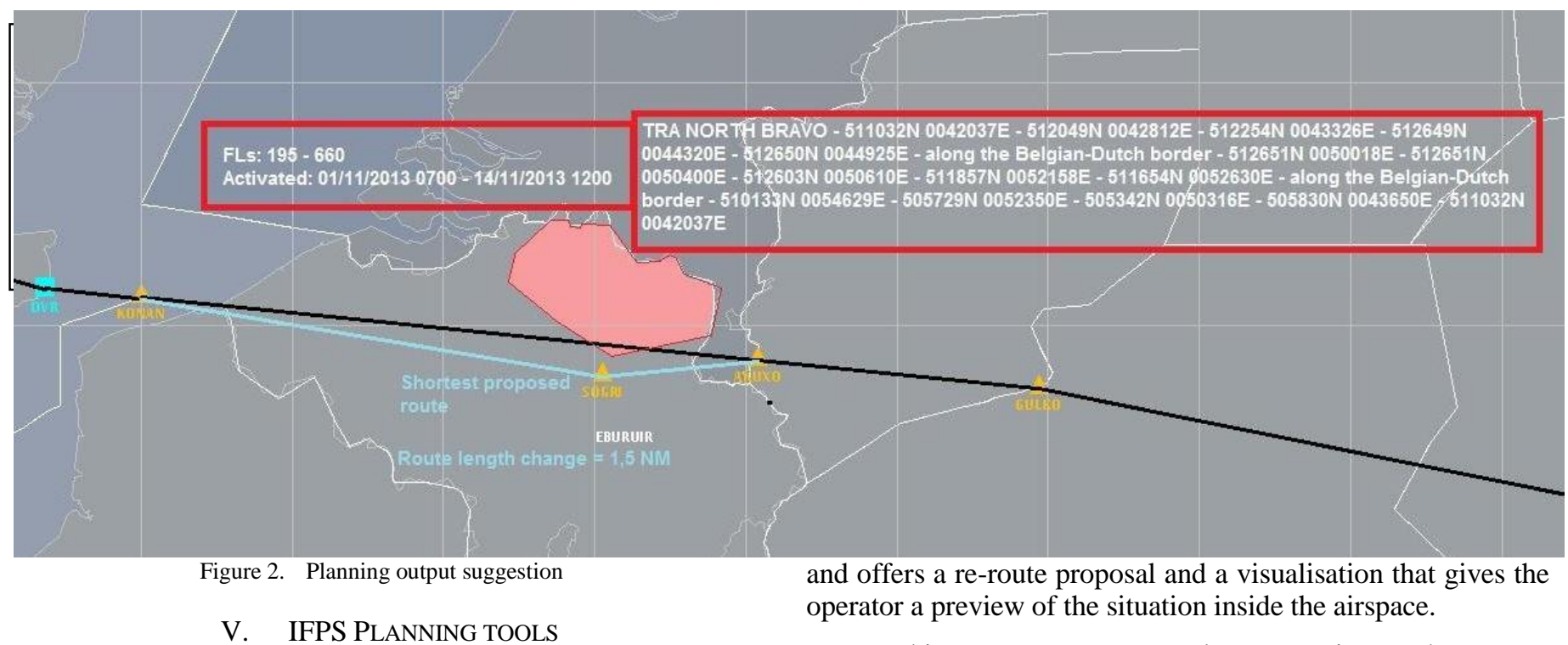

The Initial Integrated Flight Plan Processing System (IFPS) within CFMU is an existing tool that provides operators with a tool that is able to check the validity of a flight plan (FPL) on four different levels. Once the free text or structured FPL is submitted, the first level of check is a syntax check making sure that all the required fields are present in an expected format. Then a database check is taken, making sure the origin, destination and alternate aerodromes match the database as well as the aircraft type. The third check makes sure the route does not have any invalid points or that any route discontinuity is present. Most errors are usually returned in the last step, checking profile for unusable levels on a certain route or closed CDRs or that the route is RAD compliant.

In case that the submitted FPL meets all given criteria, a NO ERRORS message is shown, otherwise a list of errors
For this matter, we suggested an extension to the IFPUV Free Text Editor. This extension would expand the functionality of the tool with a "plot" button. The output of such function is seen in Fig. 2. The operator who submitted a FPL crossing an active TRA, in this case flight LKPR-EGLL crossing TRA NORTH BRAVO inside the EBUR FIR, would receive a visualised information about what portion of an airspace is the requested route crossing, information about horizontal and vertical boundaries of the airspace with an activation period. On Fig. 2, the operator-requested route is shown in black, activated TRA in red and the proposed route in blue.

The shortest suggested route by the system would be presented in a text form along with a graphical representation of the shortest possible alternative route. The route length 
change is also an important detail, therefore it is included as well.

Besides the suggested route planning tool, Fig. 2 also shows the importance of maintaining the significant point network, as it allows much more precise route alternations. A system based only on border points would never achieve such results.

\section{CONCLUSION}

In this article, we have introduced the system parts that are related to the route system and would be influenced with an introduction of Europe-wide Free Route Airspace.

We have suggested features of a flight planning tool that would show the operators all the airspace data needed to plan the flight in order to avoid activated temporary segregated and restricted areas. In the future a more proactive approach by the CFMU might be convenient for both the operators and the system, sharing airspace data and suggested routes with regards to the weather conditions and operators' requirements.

\section{REFERENCES}

[1] European Route Network Improvement Plan (ERNIP) - Framework Document - Edition June 2012 [online] [cit. 2013-11-28]. Available at $<$ http://www.eurocontrol.int/documents/ernip-framework-documentedition-june-2012>

[2] Horizontal En route Flight Efficiency - Refined methodology - Version 3.60 [online] [cit. 2013-11-28] Available at <http://prudata.webfactional.com/wiki/images/a/a9/Horizontal_En_route _Flight_Efficiency_Methodology.pdf>

[3] FLEXIBLE USE OF AIRSPACE CONDITIONAL ROUTES EUROPE [online] [cit. 2013-11-28] Available at < http://ww1.jeppesen.com/documents/aviation/publications/cdr_eur.pdf 